\title{
The Spectral Characteristics of Femtosecond Laser inscribed Long Period Grating Bend Sensors written into a Photonic Crystal Fibre
}

\author{
T. Allsop ${ }^{\text {a }}$, K. Kalli", K. Zhou, G. Smith, D.J. Webb, V. Mezentsev, I. Bennion \\ ${ }^{a}$ Photonics Research Group, Aston University, Aston Triangle, Birmingham, B4 7ET, U.K. \\ ${ }^{\#}$ Photonics Research Laboratory, Cyprus University of Technology, Cyprus \\ Telephone: $(+44) 121-359-3611$ ext. 4952 \\ Fax: $\quad(+44) 121-359-0156$ \\ email: t.d.p.allsop@aston.ac.uk
}

\begin{abstract}
A series of LPGs was inscribed in photonic crystal fibre by a low repetition femtosecond laser system. When subjected to bending they were found to be spectrally sensitive to bend orientation and displayed a strong polarisation dependence.
\end{abstract}

KEYWORDS: Photonic Crystal Fibre, Curvature, Sensing, Long Period Gratings

\section{INTRODUCTION}

A fibre long period grating (LPG) is an axially periodic refractive index variation inscribed in the core of an optical fibre. This inscription can be by ultra-violet irradiation (for photosensitive single-mode optical fibre, with an inscription mechanism dominated by photo-bleaching), fusion arc/laser heating (for non-photosensitive single-mode optical fibre, with an inscription mechanism dominated by compaction and structural change) or more recently by femtosecond lasers, where at $800 \mathrm{~nm}$ the dominating inscription mechanisms appear to be a combination of void creation, material compaction and the photo-elastic effect induced from thermal strain; these grating structures are currently under investigation by several research groups ${ }^{1,2}$. The result of the periodic refractive index variation is the coupling of light from the core of the fibre into the fibre cladding modes at discrete wavelengths. The index modulation change within the core of a single mode optical fibre can vary depending upon the inscription method used, tending to be in the range $10^{-4}$ to 0.1 , and has a period typically between $100-600 \mu \mathrm{m}$. The index modulation produces a set of attenuation bands seen in the transmission spectrum of the optical fibre core mode. The study of the LPG attenuation bands has yielded many potential applications in the field of sensing through the dependence of the position of the attenuation bands on strain $(\varepsilon)$, temperature $(T)$, the refractive index of the surrounding medium $\left(n_{s}\right)$ and bending ${ }^{3-11}$. The spectral shift of the attenuation band arises from the phase matching condition of the LPG ${ }^{1}$. Whilst the sensitivity of LPGs is an advantage over fibre Bragg gratings, cross-sensitivity between parameters can cause problems, most often an unwanted temperature sensitivity. It has been shown ${ }^{12}$ that LPGs in PCF can have very low temperature sensitivity thus reducing crosssensitivity problems, resulting in a sensing system with greater resolution.

This paper presents a study of the dependence on curvature of the spectral characteristics of LPGs written into a Photonic Crystal Fibre (PCF) using a femtosecond laser system. It was found that asymmetric (off-centre) inscription produced LPG attenuation bands that have a spectral sensitivity to the orientation of the curvature, producing red and blue wavelength shifts depending upon the bend direction and with a low temperature sensitivity. A high dependence upon the polarisation state of the illuminating light was also observed.

\section{FABRICATION OF GRATING DEVICES}

A series of LPGs with the same period, $400 \mu \mathrm{m}$, was fabricated in endlessly singlemode (ESM) fibre from Crystal Fibre $\mathrm{A} / \mathrm{S}^{13}$. Several were made with approximately the same inscription power $(0.450 \pm 0.02) \mu \mathrm{J}$, while a few others were written with different powers ranging from $0.41 \mu \mathrm{J}$ to $0.65 \mu \mathrm{J}$, requiring various grating lengths to obtain maximum strength attenuation bands. The refractive index changes to create the LPG are introduced by a femtosecond laser (800nm titanium/sapphire Spitfire/Evolution, from Spectra-Physics Lasers). The femtosecond laser radiation was focused at a predetermined point near the core with a $\times 100(\mathrm{NA}=0.55)$ microscope objective, while the fibre was moved in a direction parallel to the fibre axis. The translation speed was $100 \mu \mathrm{ms}^{-1}$. A cylindrical lens $(\mathrm{f}=100 \mathrm{~mm})$ was put in the proximity of the microscope objective to shape the laser beam at the point of inscription. The femtosecond laser system produced a $1 \mathrm{kHz}$ train of $150 \mathrm{fs}$ pulses at $800 \mathrm{~nm}$, see Figure 1; other details of the experimental set-up can be found elsewhere ${ }^{14}$. Due to previous investigations into the spectral characteristics of femtosecond laser inscribed LPGs ${ }^{15}$ the light monitoring the grating growth during fabrication was polarised; see figure $1 \mathrm{~b}$. The polarisation of the light was constantly adjusted to maximise the LPG's attenuation bands.

19th International Conference on Optical Fibre Sensors, edited by David Sampson, Stephen Collins, Kyunghwan Oh, Ryozo Yamauchi, Proc. of SPIE Vol. 7004, 70040D, (2008) 0277-786X/08/\$18 doi: 10.1117/12.786664 

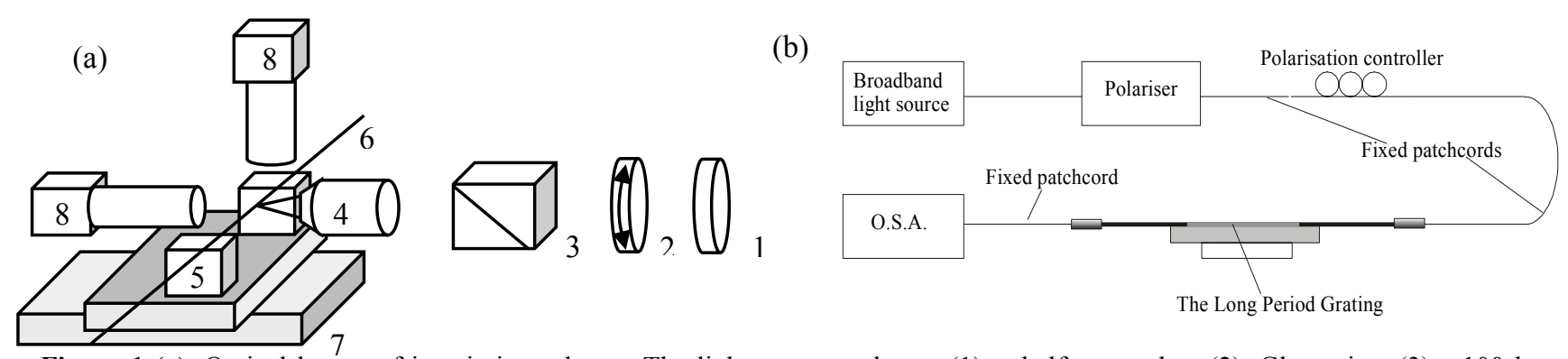

Figure 1 (a). Optical layout of inscription scheme. The light passes a shutter (1), a half-wave plate (2), Glan prism (3), x100 long working distance microscopic objective (4), before being focused in the core of the fibre (6). Two alignment 3D translation stages are mounted on top of the high precision computer controlled 2D-stage (7). We use two CCD-cameras (8) with an optical zooming system for alignment and on-line monitoring of the inscription process. (b) A schematic of the apparatus used to investigate the transmission spectra of the LPGs during fabrication.

After inscription, the LPGs were examined under a microscope and the modified regions of the fibre were identified, for an example see figure 2 .

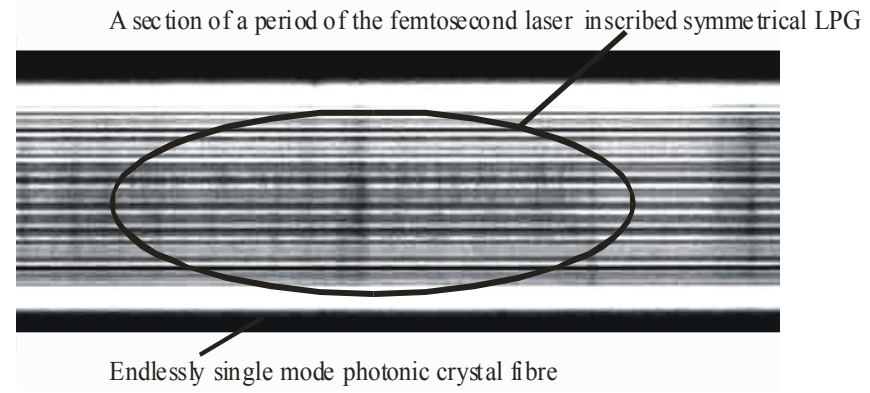

Figure 2. A photograph of part of a symmetrically inscribed section of a LPG in PCF.

Two examples of the transmission spectra of femtosecond laser inscribed LPGs are shown in figure 3; one where the structural modification of the PCF appears to be radially symmetric about the fibre axis and the other where the structural modification is asymmetric, being displaced from the fibre axis (this being achieved by translating the fibre along the optical axis of the inscribing beam). The plots show the variation of the spectra obtained by adjusting the polarisation of the illuminating light to obtain the strongest coupling response to each of the attenuation bands present in the spectrum. The variations in resonant wavelength between the two polarisation states for the attenuation band close to $1590 \mathrm{~nm}$ were $1.4 \mathrm{~nm}$ for the symmetrically written LPG and $6.4 \mathrm{~nm}$ for the asymmetric one with fiugures of $0.5 \mathrm{~nm}$ and $4.1 \mathrm{~nm}$, respectively, for the stop bands at $1350 \mathrm{~nm}$.
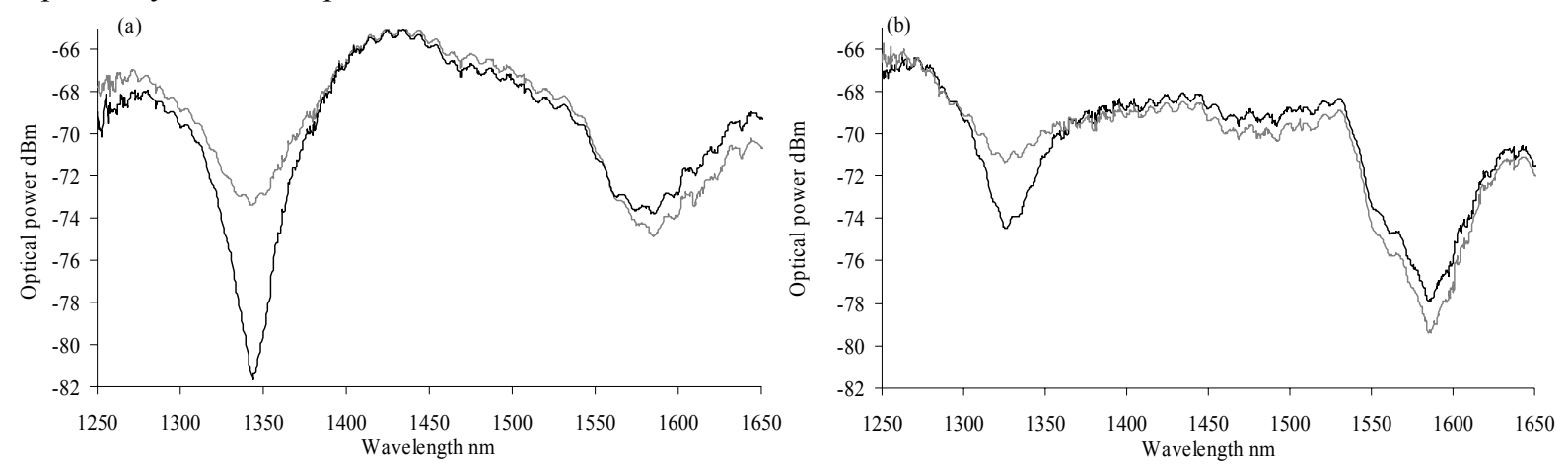

Figure 3. Examples of variation with polarisation of the transmission spectra of femtosecond laser inscribed LPGs (period $400 \mu \mathrm{m}$ ) in photonic crystal fibre (a) asymmetrical inscription (410nJ) (b) symmetrical inscription (470nJ)

\section{CURVATURE SENSITIVITY}

The LPG device was clamped between two towers; one of the clamps being mounted on a translation stage, which was moved inwards to induce a bend in the LPG placed mid-way between the clamps. Tags were used to ensure there was no twist in the fibre during the experiment and also to know the orientation of the LPG during bending, see figure 4.The 
bend sensitivities of these LPGs were investigated using a broadband light source and a polariser, which in turn was connected to a polarisation controller; the light from this arrangement illuminated the LPGs and observations of the transmitted spectrum were made using an OSA. The polarisation controller was used to maximise coupling of the illuminating light into one of attenuation bands present in the transmission spectrum of the LPG, so one attenuation band could be studied at a time

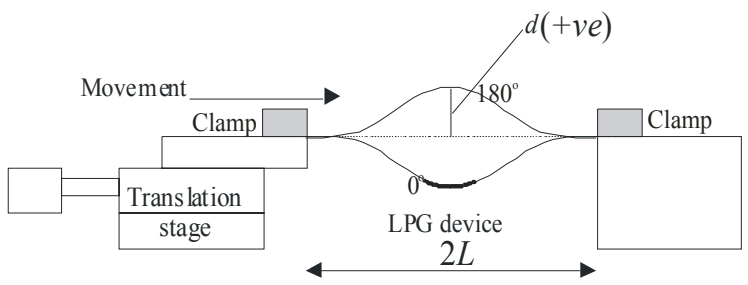

The sensor's curvature, $R$, is given by

Figure 4 Curvature test rig.

$$
R=\frac{2 \cdot d}{\left(d^{2}+L^{2}\right)}
$$

where $L$ is the half distance between the edges of the two towers and $d$ is the bending displacement at the centre of the LPG. It was found from a microscopic inspection of the LPGs that the location of the femtosecond laser induced structural modification dramatically changed the spectral sensitivity and characteristics of the LPG. The symmetrically inscribed gratings exhibited no dependence of bend sensitivity on fibre orientation (see Figure 5 for an example from the grating examined in Figure 3b), while asymmetrically inscribed LPGs did display directional bend sensitivity of some attenuation bands; the transmission spectra of such a LPG (that used for figure 3a) is shown in figure 6, and the spectral sensitivities of the two attenuation bands are shown in figure 7.
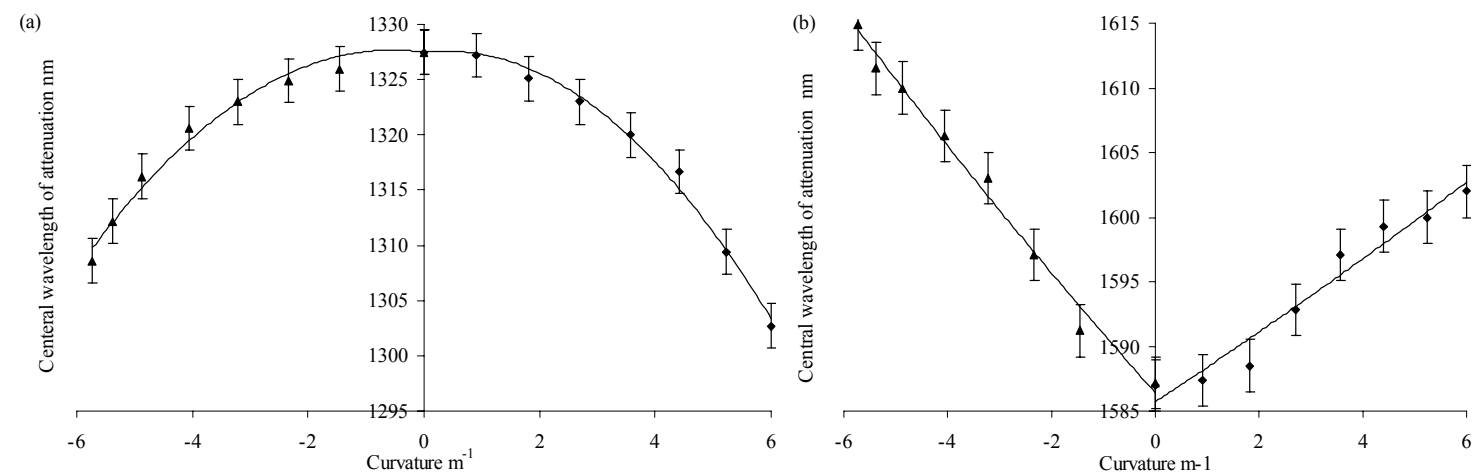

Figure 5. (a) and (b) the spectral sensitivity to curvature of both observed attenuation bands of a symmetrically inscribed LPG in ESM PCF $($ period $=400 \mathrm{~nm}$, length $=10 \mathrm{~mm}$, inscription power $=470 \mathrm{~nJ})$.
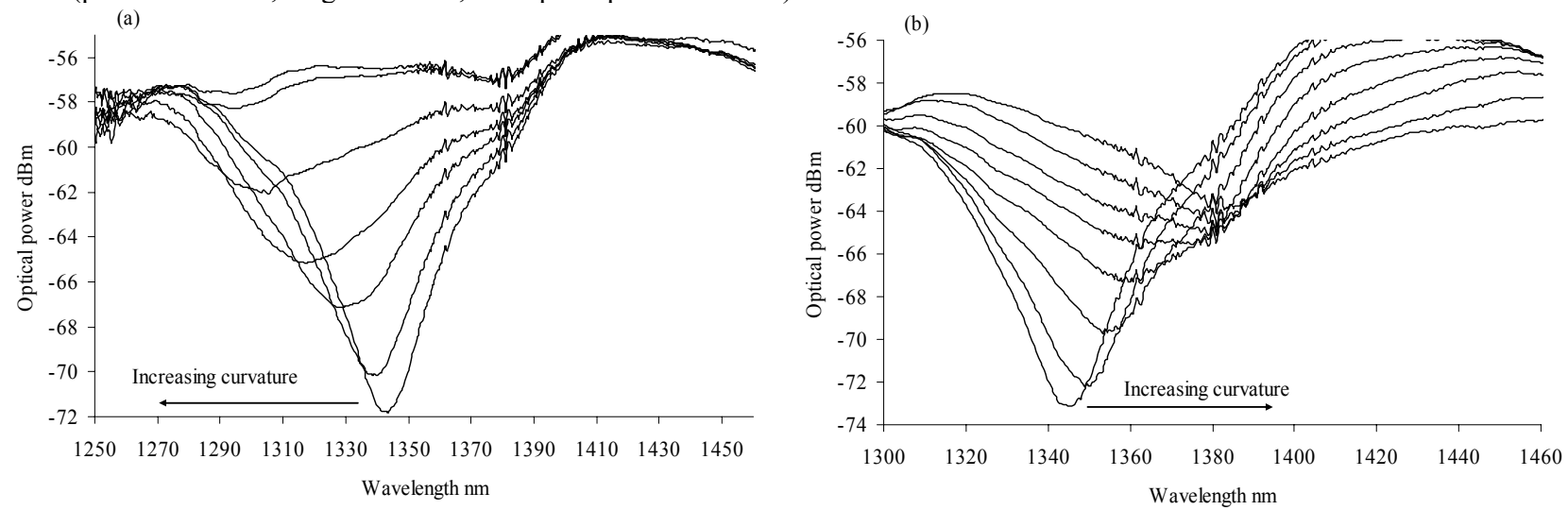

Figure 6 An example of the curvature response of the transmission spectra of an asymmetrically inscribed LPG written in ESM PCF (period $=400 \mu \mathrm{m}$, length $=96 \mathrm{~mm}$, inscription power $=410 \mathrm{~nJ})$. 

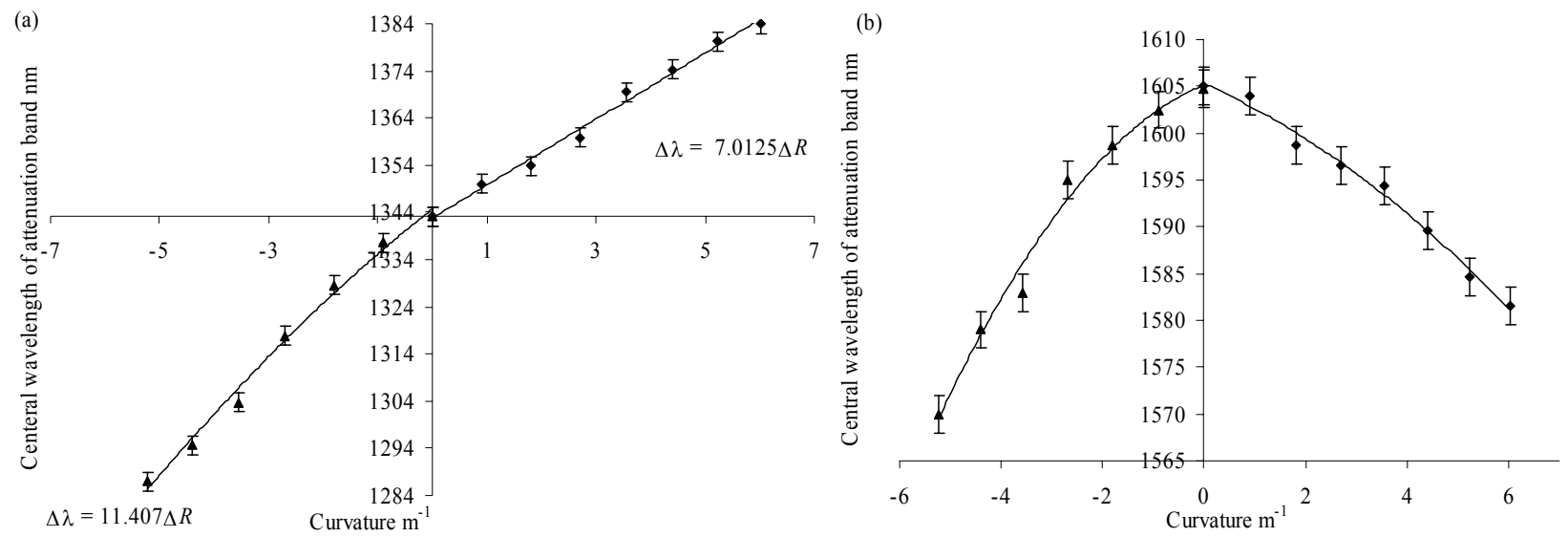

Figure 7. (a) and (b) the spectral sensitivity to curvature of both observed attenuation bands of an asymmetrically inscribed LPG in ESM PCF (period $=400 \mathrm{~nm}$, inscription power $=410 \mathrm{~nJ}$. length $=9.6 \mathrm{~mm})$.

The asymmetrically inscribed LPGs in the PCF generate some attenuation bands which display a clear directional sensitivity (Figure 7a), whilst others are much closer to giving a symmetrical response (Figure 7b). The Efield profiles of the modes in this PCF have been investigated ${ }^{14}$ and show dramatic variations in the radial distribution of the E-field with mode order, implying that we can expect radically different behaviour from the various modes.

All the LPGs exhibited polarisation dependence with spectral birefringence ranging from 1 to $10 \mathrm{~nm}$, but this appears to be mainly due to the inscription method and the power used to fabricate the LPGs (work to be published). The temperature sensitivity of these LPGs was also investigated; it was found to be $4.5 \times 10^{-3} \mathrm{~nm}^{\circ} \mathrm{C}^{-1}$ which is in approximate agreement with other workers ${ }^{14}$ and considerably less than for LPGs written in SMF ${ }^{6,15}$.

\section{CONCLUSION}

A series of LPGs was inscribed in photonic crystal fibre by a femtosecond laser system; when subjected to bending they were found to be spectrally sensitive to bend orientation, yielding red and blue wavelength shifts depending upon fibre curvature with a low temperature sensitivity. This suggests that these PCF devices have the potential to be applied as curvature sensors with high resolution.

\section{References}

1 D. Linde et al, "Breakdown threshold and plasma formation in femtosecond laser-solid interaction", JO SA. B, Vol. 13, No. 1, pp.216, 1996

2 A. Streltsov et al, "Study of femtosecond-laser-written waveguides in glasses", JOSA B, Vol. 19, No. 10, pp. 2496-2504, 2002

3 A. Vengsarkar et al, "Long-Period Gratings as Band-Rejection Filters", J. Lightwave Technol., vol.14, no.1, pp.58-64, 1996.

4 V. Bhatia, A.M. Vengsarkar, "Optical fibre long-period grating sensors", Opt. Lett., vol.21, no.9, pp.692-694, 1996.

5 H.J. Patrick, A.D. Kersey, F. Bucholtz, "Analysis of the response of long period fiber gratings to external index of refraction", J. Lightwave Technol., vol.16, no.9, pp.1606-1612, 1998.

6 V. Bhatia, "Applications of long-period gratings to single and multi-parameter sensing", Opt. Express., 1999, vol. 4, no. 11, pp.457-466.

$7 \mathrm{X}$. Shu et al, "Room-temperature operation of widely tunable loss filter", Electron. Letts. vol.37, no. 4, pp.216-218, 2001.

8 T. Allsop et al, " Detection of Organic Aromatic compounds in Paraffin by a long period fiber grating optical sensor with optimised sensitivity", Opt. Commun., vol.191, pp.181-190, 2001.

9 H.J. Patrick, C.C. Chang, S.T. Vohra, "Long period fibre gratings for structural bend Sensing”, Electron. Letts., vol. 34, no. 18, pp. 1773, 1998.

10 W. Du, H. Tam, M. Liu, X. Tao, "Long-period fiber grating bending sensors in laminated composite structures," in Sensory Phenomena and Measurement Instrumentation for Smart structures and materials. Proc. SPIE 3330, pp.284-292, 1998.

11 H.J. Patrick, "Self-aligning, bipolar bend transducer based on long period grating written in eccentric core fibre", Electron. Letts., vol. 36, no. 21 , p. $1763-1764,2000$.

12 Endlless Single Mode ESM-12-01 ${ }^{\mathrm{TM}}$, CRYSTAL FIBRE A/S, “http://www.crystal-fibre.com/”

13 A. Martinez, et al, "Point by point FBG inscription by a focused NIR femtosecond laser", OSA Conf.Proc. CLEO/IQEC and PhAST Washington, DC, 2004, CMY6

14 J. Petrovic et al, "Sensitivity of LPGs in PCFs Fabricated by an Electric Arc to Temperature, Strain, and External Refractive Index", J. Lightwave Technol, Vol.25, no.5, pp. 1306-1312

15 T. Allsop, et al, "A comparison of the spectral properties of high temperature annealed long period gratings inscribed by fs laser, UV, and fusion arc", SPIE Vol 6193, Photonics Europe, Strasburg, France, 2006. 